\title{
ESTUDO DA INFLUÊNCIA DA MASSA DE ESCÓRIA E DE BANHO NA DESFOSFORAÇÃO DE FERRO-GUSA COM A TERMODINÂMICA COMPUTACIONAL*
}

Anna Paula Littig Berger ${ }^{1}$ Daniela Bahiense de Oliveira² Cynara Christ Klippe/ ${ }^{3}$ Camila Santos Scope/ 4 José Roberto de Oliveira ${ }^{5}$

\section{Resumo}

A remoção do fosfóro através do processo de desfosforação do ferro-gusa tem sido cada vez mais relevante, devido a influência que exerce de forma prejudicial as propriedades do aço e devido a extensão do cenário de escassez de minérios com menor teores de fosfóro. Este trabalho tem como objetivo a avaliação termodinâmica computacional de misturas a base de dolomita e finos de minério utilizadas no processo de desfosforação realizado em panela para o refino do ferro-gusa. Foi utilizado o software Factsage para avaliação termodinâmica, tendo este se mostrado eficaz na determinação da concentração de fósforo de equilíbrio no metal, fases presentes e temperatura de fusão das misturas. Verificou-se uma tendência de maiores valores de eficiência para o processo de desfosforação quando aumenta-se a razão de massa de desfosforante e massa de gusa.

Palavras-chave: Pré-tratamento de ferro-gusa; Desfosforação; Termodinâmica computacional.

\section{THERMODYNAMIC COMPUTATIONAL STUDY OF THE INFLUENCY EFFECT OF SLAG AND BATH MASS IN HOT METAL DEPHOSPHORIZATION}

\begin{abstract}
The removal of phosphorus through hot metal dephosphorization process has been increasingly important, since the injurious influences of phosphorus on the properties of steel and due the cenary extension of scarce ore with lower phosphorus. This work aims to computational thermodynamic evaluation of mixtures based on dolomite and ore fines used in the process of pot hot metal dephosphorization. It was used the FactSage software for thermodynamic evaluation, since it has showed effective in determining the equilibrium phosphorus concentration in the metal, present phases, and melting temperature of the mixtures. Was possible to verify a tendency of major values for dephosphorization efficiency when increases the ratio between dephosphor mass and hot metal mass.

Keywords: Hot metal; Pre-treatment; Dephosphorization; Computational thermodynamics.

\footnotetext{
Engenheira Metalurgista e Mestranda em Engenharia Metalúrgica e de Materiais. Graduanda em Engenharia Metalúrgica e de Materiais.

Engenheira Metalurgista e Mestranda em Engenharia Metalúrgica e de Materiais.

Graduanda em Engenharia Metalúrgica, Instituto Federal do Espírito Santo, Vitória, ES, Brasil.

Prof. Dr. do Instituto Federal do Espírito Santo, IFES, dos cursos de graduação e pós-graduação em Engenharia Metalúrgica e de Materiais.
} 


\section{INTRODUÇÃO}

Inúmeras modificações e avanços tecnológicos foram e tem sido feitos a fim de aprimorar o processo de fabricação de aço para obtê-lo com maior limpidez, com velocidade de produção e o menor custo possível. ${ }^{1}$

A presença de fósforo no metal afeta adversamente algumas propriedades do aço como a tenacidade e a resistência. Além disso, a presença de fósforo causa a segregação intergranular durante o lingotamento contínuo e deteriora a ductilidade e resistência de produtos como tubos, aços criogênicos e placas de vasos de pressão².

A etapa de desfosforação de ferro-gusa pode ser realizada no carro torpedo, na panela de transferência de gusa ou até mesmo em um convertedor específico para este fim. Quando a DeP é realizada no convertedor, esta ocorre sem que haja uma redução do teor de carbono, e com duas etapas de vazamento de escória. Este procedimento é o que confere melhores resultado na DeP. ${ }^{1}$

Empresas ao redor do mundo implantaram e tem desenvolvido tecnologias de prétratamento de ferro-gusa. Entretanto, cada empresa adota um sistema de prétratamento adequado aos seus equipamentos existentes, layout e condições operacionais, onde as etapas de dessiliciação, dessulfuração e desfosforação são realizadas em equipamentos específicos para estes processos.

Algumas usinas japonesas já incorporaram etapas para redução do silício e do fósforo como operações de refino prévio do ferro-gusa, assim como a Siderúrgica Ibiraçu.

Com as exigências de teores de fósforo nos aços cada vez menores, e os teores de fósforo nos minérios de ferro cada vez maiores, os processos de desfosforação (DeP) de ferro-gusa ganham cada vez mais força.

Existem diferentes parâmetros da literatura para se avaliar termodinamicamente o processo de desfosforação de ferro-gusa, tais como o coeficiente de partição do fósforo (LP), a capacidade de fosfato $\left(C_{P}\right)$ e a basicidade ótica $(\Lambda)$.

Por definição o coeficiente de partição do fósforo (Lp) é a razão no equilíbrio da porcentagem de fósforo presente na escória $(\% \mathrm{Peq})$ e a porcentagem de fósforo presente no metal [\%Peq], conforme a equação 1.

$$
\mathrm{Lp}=\frac{\left(\% \mathrm{P}_{\mathrm{eq}}\right)}{\left[\% \mathrm{P}_{\mathrm{eq}}\right]}(1)
$$

Entretanto a reação de desfosforação ocorre na interface metal e escória e não ocorre no equilíbrio, dificultando a real medição desses valores. Por isso vários autores estudaram e definiram empiricamente equações que pudessem medir os valores do coeficiente de partição do fósforo, que podem ser visualizados na Tabela 1.

Tabela 1. Modelos termodinâmicos da literatura utilizados.

\begin{tabular}{|c|c|}
\hline Autor & Modelo \\
\hline Young & $\begin{array}{c}\log C p=-18,184+35,84 \cdot \Lambda-22,35 \cdot \Lambda^{2}+\frac{22 \cdot 930 \cdot \Lambda}{T}-0,06257 . \% \mathrm{FeO} \\
-0,04256 . \% \mathrm{MnO}+0,359 . \% \mathrm{P}_{2} O_{5}^{0,3}\end{array}$ \\
\hline Healy & $\log \frac{(\% P)}{[\% P]}=\frac{22.350}{T}+0,08 \cdot(\% C a O)+2,5 \cdot \log \left(\% F e_{\text {total }}\right)-16$ \\
\hline $\begin{array}{l}\text { Suito e } \\
\text { Inoue }\end{array}$ & $\log \frac{(\% P)}{[\% P]}=0,072[(\% C a O)+0,3 \cdot(\% M g O)]+2,5 \cdot \log \left(\% F e_{\text {total }}\right)+\frac{11 \cdot 570}{T}-10,52$ \\
\hline Turkdogan & $\log \left(\gamma_{P_{2} O_{5}}\right)=-1,12 \cdot\left(22 \cdot N_{C a O}+15 \cdot N_{M g O}+13 \cdot N_{M n O}+12 \cdot N_{F e O}-2 \cdot N_{S i O 2}\right)-\frac{42000}{T}+23,58$ \\
\hline
\end{tabular}


A remoção do fósforo do metal e a consequente fixação do óxido formado na escória dependem da atividade do $\mathrm{P}_{2} \mathrm{O}_{5}$ na escória, sendo esta em função da composição química e temperatura. Quanto maior a concentração de $\mathrm{P}_{2} \mathrm{O}_{5}$ na escória, maior será a concentração de fósforo no metal, conforme equação 2. Sendo Np205 a fração molar do composto.

A concentração de $\mathrm{P}_{2} \mathrm{O}_{5}$ na escória está diretamente relacionada a sua atividade na mesma, conforme é mostrado a seguir.

Turkdogan (1996) descreve a variação do coeficiente de atividade do $\mathrm{P}_{2} \mathrm{O}_{5}$ com a temperatura e composição da escória em uma relação empírica, mostrada na Tabela 1 .

$$
\boldsymbol{a}_{P_{2} o_{5}}=N_{P_{2} o_{5}} \cdot \gamma_{P_{2} o_{5}}(2)
$$

A basicidade de cada óxido é calculada em função da eletronegatividade de Pauling e a basicidade teórica da escória é dada pela média ponderada das contribuições individuais, através da fração molar, conforme Equação 3 e Equação 4:

$$
\begin{gathered}
\Lambda_{i}=\frac{0,74}{(X-0,26)} \\
\Lambda_{e s c}=\sum \Lambda_{i} N
\end{gathered}
$$

Onde $\mathrm{X}$ é a eletronegatividade de Pauling, $\mathrm{N}$ fração molar do óxido ou fluoreto na escória, $\Lambda_{i}$ basicidade de cada óxido e $\Lambda_{e s c}$ basicidade da escória.

O Fator de Desfosforação (F DeP ) correlaciona as informações obtidas através das simulações computacionais e a eficiência encontrada na prática, levando em consideração a fração de fase líquida formada na escória na temperatura de $1400^{\circ} \mathrm{C}$, a atividade do $\mathrm{CaO}$ e a atividade do FeO. Este fator é definido conforme a equação 5 , onde a \%Líquido/100 e a \%Sólido/100 podem ser representadas também como NLíquido e Nsólido (fração mássica de líquido e fração mássica de sólido), respectivamente.

$$
\text { Fator }_{\text {DeP }}=a_{\text {CaO }}+a_{\text {FeO }}+\left(N_{\text {Liquido }}-N_{\text {Solido }}\right)
$$

Cada vez mais se torna necessário, tendo em vista as características das reações de desfosforação do ferro-gusa, as simulações de melhores adições e das condições termodinâmicas favoráveis a essas reações. Essas simulações contribuem de forma a diminuir número de testes experimentais e do tempo gasto para a análise de misturas aplicadas ao pré-tratamento de ferro-gusa, tendo como consequência uma redução de custos.

\section{MATERIAIS E MÉTODOS}

Para a realização do presente trabalho foram realizadas as seguintes etapas:

- Caracterização dos materiais usados nas corridas preliminares da Siderúrgica de Ibiraçu;

- Composição da mistura;

- Realização dos cálculos termodinâmicos;

- Avaliação das misturas através dos softwares de termodinâmica computacional.

\subsection{Caracterização dos Materiais}

Como base para o início dos estudos e aplicação dos conceitos técnicos foram feitas corridas com o procedimento padrão da Siderúrgica de lbiraçu.

Ao término da redução do minério de ferro no alto forno, o ferro-gusa é vazado do para panela, onde será realizado o processo de desfosforação. Nesta etapa realizase uma primeira separação da escória do ferro-gusa. 
Após o canal de corrida o ferro-gusa é vazado na panela de tratamento. No início do vazamento começa a injeção de nitrogênio pela parte inferior da panela. Quando o ferro-gusa cobre todo o fundo da panela, é iniciada a adição de mistura desfosforante. Essa mistura desfosforante é composta de uma relação mássica de $1: 1$ de dolomita e finos de minério de ferro.

Após o término do carregamento do ferro-gusa na panela, inicia-se o sopro de oxigênio. Posteriormente ao término do sopro de oxigênio, a lança é retirada, e em seguida uma amostra do ferro-gusa é coletada para análise química.

Após iniciado o basculamento da panela para o vazamento do ferro-gusa tratado na máquina de moldar, uma amostra do ferro-gusa é coletada para análise química, e durante o vazamento é retirado à escória. A temperatura do processo se manteve em torno de $1370^{\circ} \mathrm{C}$.

Após a verificação dos procedimentos executados para o pré-tratamento da Siderúrgica de lbiraçu, foram coletadas amostras de 12 corridas, com as análises dos teores de $\mathrm{Si}, \mathrm{Mn}, \mathrm{P}, \mathrm{S}$ e $\mathrm{C}$ antes do pré tratamento na panela e após. Estes resultados estão apresentados na Tabela 2.

Tabela 2. Análise das corridas preliminares antes e após do tratamento e massa de desfosforante e massa de gusa utilizadas.

\begin{tabular}{|c|c|c|c|c|c|c|c|c|c|c|c|c|}
\hline \multirow{2}{*}{ Corrida } & \multicolumn{2}{|c|}{$\% \mathrm{Si}$} & \multicolumn{2}{|c|}{$\% \mathrm{Mn}$} & \multicolumn{2}{|c|}{$\% \mathrm{P}$} & \multicolumn{2}{|c|}{$\% \mathrm{~S}$} & \multicolumn{2}{|c|}{$\% \mathrm{C}$} & \multirow{2}{*}{$\begin{array}{c}\text { Massa } \\
\text { desfosforante } \\
(\mathrm{kg})\end{array}$} & \multirow{2}{*}{$\begin{array}{l}\text { Massa de } \\
\text { gusa }(\mathrm{kg})\end{array}$} \\
\hline & inicial & final & inicial & final & inicial & final & inicial & final & inicial & final & & \\
\hline M1 & 0,22 & 0,1 & 0,066 & 0,02 & 0,076 & 0,02 & 0,019 & 0,01 & 4,37 & 4,3 & 1250 & 19280 \\
\hline M2 & 0,2 & 0,1 & 0,06 & 0,03 & 0,074 & 0,03 & 0,014 & 0,01 & 4,35 & 4,4 & 1250 & 19820 \\
\hline M3 & 0,18 & 0,1 & 0,064 & 0,01 & 0,077 & 0,03 & 0,017 & 0,01 & 4,38 & 4,5 & 1000 & 16950 \\
\hline M4 & 0,14 & 0,1 & 0,053 & 0,01 & 0,073 & 0,02 & 0,019 & 0,01 & 4,32 & 4,4 & 1000 & 23440 \\
\hline M5 & 0,16 & 0,1 & 0,06 & 0,01 & 0,07 & 0,03 & 0,022 & 0,01 & 4,39 & 4,4 & 750 & 19650 \\
\hline M6 & 0,1 & 0,1 & 0,065 & 0,01 & 0,076 & 0,03 & 0,017 & 0,01 & 4,36 & 4,5 & 750 & 23470 \\
\hline M7 & 0,08 & 0,1 & 0,048 & 0,01 & 0,078 & 0,02 & 0,014 & 0,01 & 4,3 & 4,4 & 1000 & 20490 \\
\hline M8 & 0,15 & 0,1 & 0,055 & 0,03 & 0,075 & 0,03 & 0,017 & 0,01 & 4,38 & 4,2 & 500 & 24320 \\
\hline M9 & 0,16 & 0,1 & 0,05 & 0,03 & 0,072 & 0,03 & 0,014 & 0,01 & 4,34 & 4,3 & 750 & 22110 \\
\hline M10 & 0,23 & 0,1 & 0,066 & 0,02 & 0,071 & 0,03 & 0,01 & 0,01 & 4,38 & 4,4 & 750 & 22040 \\
\hline M11 & 0,2 & 0,1 & 0,062 & 0,03 & 0,078 & 0,05 & 0,017 & 0,01 & 4,35 & 4,4 & 500 & 21860 \\
\hline M12 & 0,22 & 0,1 & 0,066 & 0,03 & 0,074 & 0,04 & 0,01 & 0,01 & 4,34 & 4,4 & 750 & 19730 \\
\hline
\end{tabular}

\subsection{Caracterização das Misturas}

No presente trabalho como a mistura usada foi na proporção de 1:1 entre a dolomita e os finos de minério, foi criada a Tabela 3 que mostra a composição da mistura, quando usado dolomita já no banho, ou seja, considerando as calcinações. 
Tabela 3. Composição da mistura no banho

\begin{tabular}{cc}
\hline COMPONENTE & \% Componente no banho \\
\hline $\mathrm{FeO}$ & $65,98 \%$ \\
$\mathrm{CaO}$ & $19.99 \%$ \\
$\mathrm{MgO}$ & $8,27 \%$ \\
$\mathrm{SiO}_{2}$ & $1.89 \%$ \\
$\mathrm{Al}_{2} \mathrm{O}_{3}$ & $0,48 \%$ \\
$\mathrm{P}$ & $0,007 \%$ \\
$\mathrm{Mn}$ & $0,015 \%$ \\
$\mathrm{TiO}_{2}$ & $0,04 \%$ \\
$\mathrm{PPC}$ & $0,045 \%$ \\
$\mathrm{H}_{2} \mathrm{O}$ & $1,22 \%$ \\
$\mathrm{Outros}$ & $2,04 \%$ \\
\hline
\end{tabular}

Os resultados apresentados na Tabela 4, se mostram propícios ao processo de desfosforação apresentado pela Equação 6, devido aos teores de $\mathrm{FeO}$ e $\mathrm{CaO}$ aferidos.

$$
2 \underline{P}+5(\mathrm{FeO})+3(\mathrm{CaO})=\left(3 \mathrm{CaO} \cdot P_{2} \mathrm{O}_{5}\right)_{(s)}+5 \underline{F e}
$$

Foi utilizado o software Factsage para determinar a quantidade de líquido e fases sólidas formadas nas misturas propostas no presente trabalho.

Foram utilizados os bancos de dados FactPS e FtOxid disponíveis nesse software. O primeiro atua na determinação dos compostos sólidos formados e o segundo atua na avaliação dos compostos formados na fase líquida da mistura/escória. Foram usados Pure Solids e Slag? do sub-banco de dados existente.

\subsection{Determinação das condições de equilíbrio termodinâmico da reação de desfosforação}

Foi utilizado o software Factsage para avaliar as condições de equilíbrio termodinâmico da reação de desfosforação. O banco de dados do software em questão utilizado para determinar as condições de equilíbrio do ferro-gusa foi o FSstel. Os bancos de dados FactPS e FtOxid atuaram na determinação das fases formadas na escória gerada no equilíbrio termodinâmico.

\section{RESULTADOS E DISCUSSÃO}

\subsection{Avaliação da eficiência da desfosforação através das misturas iniciais}

Para uma verificação dos resultados apresentados na Tabela 2 foi criada a Figura 1, a qual mostra para cada um dos parâmetros analisados ( $\mathrm{P}, \mathrm{Si}, \mathrm{S}$ e $\mathrm{Mn}$ ), seu comportamento em relação ao tratamento em panela.

As Figuras 1 e 2 apresentam os valores da fração mássica inicial e final dos elementos analisados. Para se obter estes valores foi feito uma média aritmética das frações dos componentes para cada grupo de massa de desfosforante utilizada $(500,750,1000$ e $1250 \mathrm{~kg})$, e foi desconsiderado a massa de gusa. 

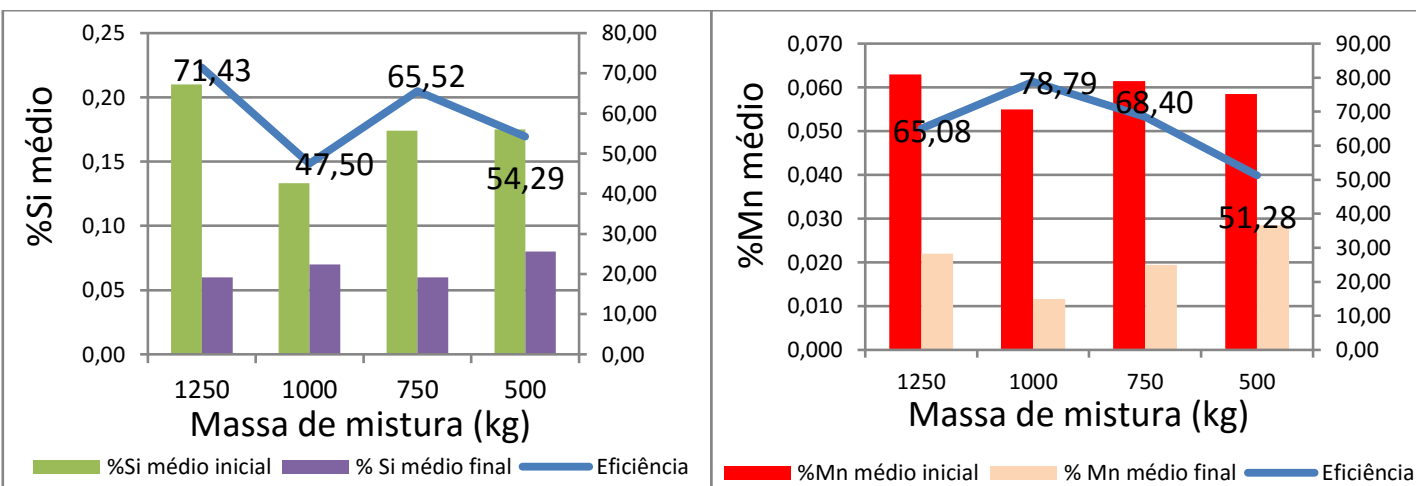

Figura 1. Redução média dos teores de (a) Si e (b) Mn em relação a massa de desfosforante aplicada.
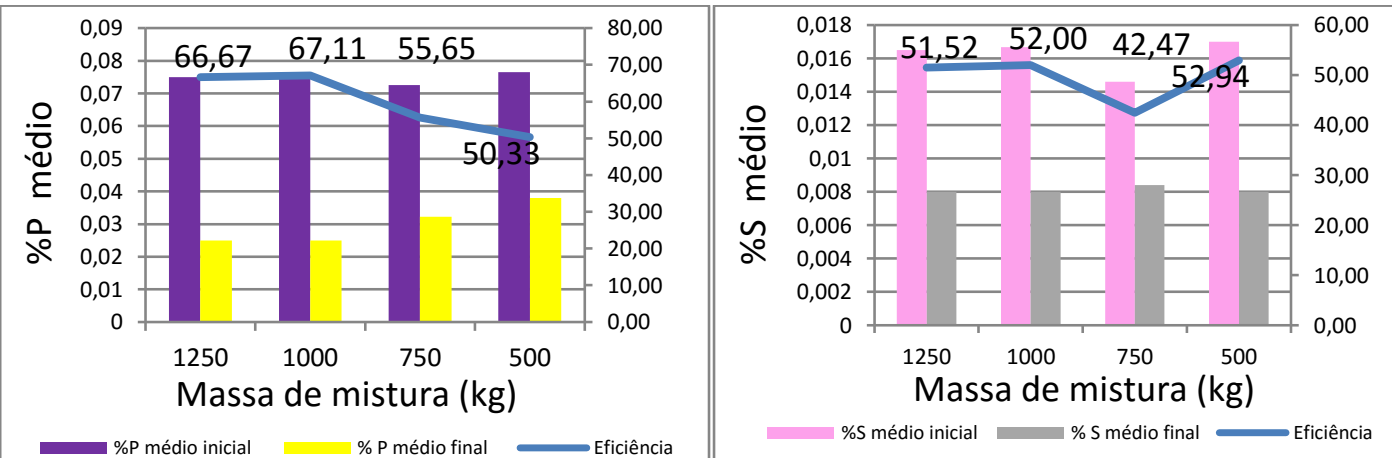

Figura 2. Redução média dos teores de (a) P e (b) S em relação a massa de desfosforante aplicada.

Pode-se observar pelas Figuras 1 e 2 que as massas de mistura de $1000 \mathrm{~kg}$ e 1250 $\mathrm{kg}$ apresentaram bons resultados de redução média dos teores de Si, Mn, P e S.

Em análise preliminar destes dados, pode-se concluir inicialmente que a massa ideal de desfosforante estaria entre 1000 e $1250 \mathrm{~kg}$, e para melhor análise foi criada a Tabela 4. A Figura 3 apresenta o comportamento dos teores de P, Si, S e Mn no metal em relação a massa de desfosforante por tonelada de gusa aplicado na corrida.

Tabela 4. Dados das corridas.

\begin{tabular}{ccccc}
\hline Corrida & $\begin{array}{c}\text { Desf. } \\
\text { (kg) }\end{array}$ & $\begin{array}{c}\text { Gusa } \\
(\mathbf{k g})\end{array}$ & $\begin{array}{c}\text { Massa de } \\
\text { DeP/Massa } \\
\text { de gusa } \\
\text { (kg/ton) }\end{array}$ & $\eta \%$ \\
\hline 8 & 500 & 24.320 & 20,56 & 60 \\
\hline 11 & 500 & 21.860 & 22,87 & 41 \\
\hline 12 & 750 & 19.730 & 25,34 & 51,4 \\
\hline 6 & 750 & 23.470 & 31,96 & 60,5 \\
\hline 9 & 750 & 22.110 & 33,92 & 52,8 \\
\hline 10 & 750 & 22.040 & 34,03 & 54,9 \\
\hline 5 & 750 & 19.650 & 38,17 & 61,4 \\
\hline 4 & 1000 & 23.440 & 42,66 & 67,1 \\
\hline 7 & 1000 & 20.490 & 48,8 & 69,2 \\
\hline 3 & 1000 & 16.950 & 59.00 & 64,9 \\
\hline 2 & 1250 & 19.820 & 63,07 & 63,5 \\
\hline 1 & 1250 & 19.280 & 64,83 & 69,7 \\
\hline & & & &
\end{tabular}
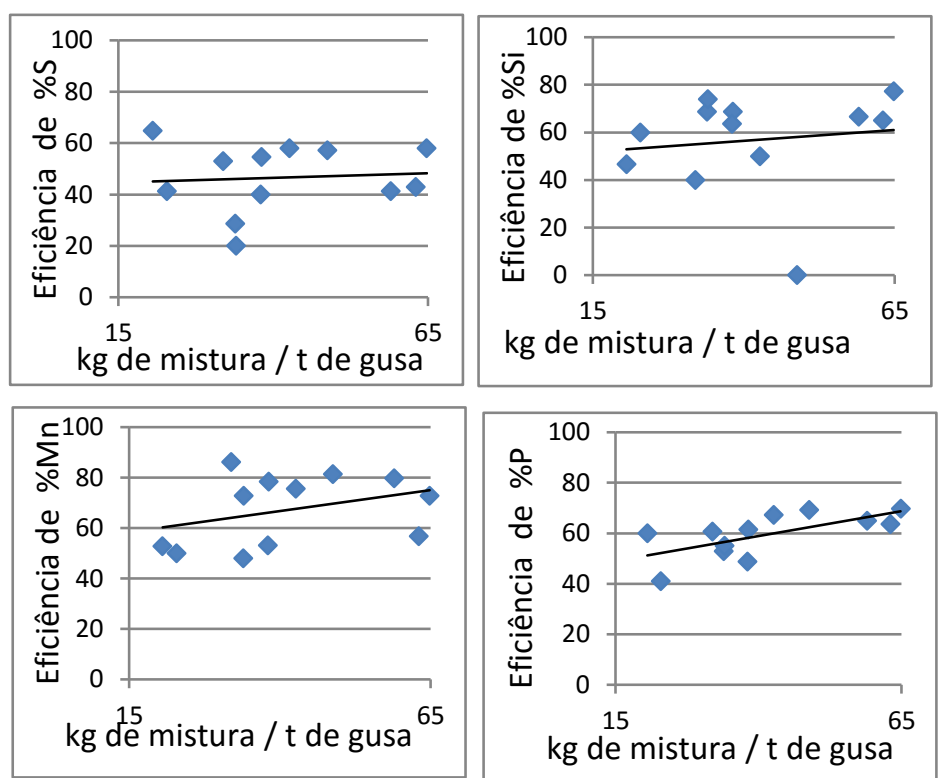

Figura 3. Comportamento dos teores de P, Si, S e Mn. 
Observa-se que na Figura 3 o comportamento do teor de P, Si e Mn final no metal em relação à massa de desfosforante por tonelada de gusa aplicado na corrida, na qual verifica-se uma tendência de maior eficiência quando aumentamos a massa de desfosforante em relação à massa de gusa.

Para o S não se verifica esta tendência tão acentuada de maior ou menor eficiência quando aumentamos a massa de desfosforante em relação à massa de gusa, o que pode ser resultado da falta de controle da temperatura em cada corrida.

\subsection{Avaliação termodinâmica com modelos literários}

A análise termodinâmica da desfosforação do ferro-gusa foi feita através dos modelos apresentados na Introdução, sendo a basicidade ótica, a capacidade de fosfato e o coeficiente de partição do fósforo. A capacidade de fosfato foi calculada a partir do modelo de Young (1991), o coeficiente de partição do fósforo foi calculado a partir dos modelos de Healy (1970) e Suito e Inoue (2006), e o coeficiente de atividade do $\mathrm{P}_{2} \mathrm{O}_{5}$ pelo modelo de Turkdogan (2000).

Tabela 5. Condições termodinâmicas do processo de desfosforação

\begin{tabular}{ccccc}
\hline$\Lambda$ & $\begin{array}{c}\log (\mathrm{CP}) \\
\text { Young }\end{array}$ & $\begin{array}{c}\text { Log (LP) } \\
\text { Healy }\end{array}$ & $\begin{array}{c}\text { Log (LP) } \\
\text { Suito }\end{array}$ & х P2O5 \\
\hline 0,663 & 0,873 & 3,477 & 2,415 & $7,18 \mathrm{E}-19$ \\
\hline
\end{tabular}

Devido ao fato de que as misturas possuem a mesma composição, os valores dos parâmetros acima calculados são iguais para M1 a M12 independente da massa da mistura desfosforante, uma vez que esses parâmetros levam em consideração fração molar ou porcentagem dos componentes da mistura.

Contudo através da literatura observa-se que esses parâmetros não são precisos para prever a eficiência do processo de desfosforação uma vez que se tratam do equilíbrio termodinâmico. E a partir dos dados apresentados na Tabela 6 , não é possível relacionar diretamente a eficiência do processo de desfosforação com o aumento da basicidade ótica, a diminuição do coeficiente de atividade do $\mathrm{P}_{2} \mathrm{O}_{5}, 0$ aumento da capacidade de fosfato ou ainda do coeficiente de partição de fósforo da escória final.

De acordo com a literatura, quanto maior o coeficiente de partição, maior a eficiência do processo. Os valores encontrados não se mostram bons para melhor eficiência do processo de desfosforação quando comparados com a literatura. [1]

A equação 2 mostra que quanto menor o coeficiente de atividade de $\mathrm{P}_{2} \mathrm{O}_{5}$, menor será sua atividade e, assim, menor será a concentração de fósforo no metal. Da literatura, pode-se observar então que o valor obtido de coeficiente de $\mathrm{P}_{2} \mathrm{O}_{5}$ para as misturas é um bom valor. [1]

\subsection{Avaliação através da Termodinâmica computacional}

Por meio do software FactSage, foi determinado as condições de equilíbrio do metal e das escórias assim como as possíveis fases presentes para cada uma das misturas, sendo os resultados apresentados na Tabela 6 e Tabela 7. Foi calculado o Fator de Desfosforação pela equação 5. 
Tabela 6. Dados fornecidos pelo Software Factsage.

\begin{tabular}{cccccccc}
\hline Corrida & $\begin{array}{c}\text { Mass de } \\
\text { desfosforante } \\
(\mathrm{kg})\end{array}$ & $\begin{array}{c}\text { Massa } \\
\text { de gusa } \\
(\mathrm{kg})\end{array}$ & Desf./gusa $(\mathrm{kg} / \mathrm{t})$ & Pinicial & Pfinal & $\%$ Peq & $\eta \%$ \\
\hline M2 & 1250 & 19280 & 64,83 & 0,076 & 0,023 & $8,88 \mathrm{E}-05$ & 69,74 \\
\hline M3 & 1000 & 20490 & 61,01 & 0,078 & 0,024 & $5,20 \mathrm{E}-05$ & 69,23 \\
\hline M7 & 1000 & 23440 & 42,66 & 0,073 & 0,024 & $7,54 \mathrm{E}-05$ & 67,12 \\
\hline M4 & 1000 & 16950 & 59,00 & 0,077 & 0,027 & $1,00 \mathrm{E}-04$ & 64,94 \\
\hline M1 & 1250 & 19820 & 37,84 & 0,074 & 0,027 & $7,94 \mathrm{E}-05$ & 63,51 \\
\hline M12 & 750 & 19650 & 38,17 & 0,07 & 0,027 & $1,46 \mathrm{E}-04$ & 61,43 \\
\hline M6 & 750 & 23470 & 42,61 & 0,076 & 0,03 & $8,30 \mathrm{E}-05$ & 60,53 \\
\hline M8 & 500 & 24320 & 20,56 & 0,075 & 0,03 & $7,31 \mathrm{E}-04$ & 60 \\
\hline M10 & 750 & 22040 & 34,03 & 0,071 & 0,032 & $3,67 \mathrm{E}-04$ & 54,93 \\
\hline M9 & 750 & 22110 & 33,92 & 0,072 & 0,034 & $1,53 \mathrm{E}-04$ & 52,78 \\
\hline M5 & 750 & 19730 & 25,34 & 0,074 & 0,038 & $3,39 \mathrm{E}-04$ & 48,65 \\
\hline M11 & 500 & 21860 & 34,31 & 0,078 & 0,046 & $1,12 \mathrm{E}-02$ & 41,03 \\
\hline
\end{tabular}

Tabela 7. Dados das fases presentes no equilíbrio

\begin{tabular}{ccccc}
\hline \%Liquido & \%Sólido & aCaO & aFeO & F DeP \\
\hline 94,11 & 5,89 & 0,316 & 0,645 & 2,31 \\
\hline
\end{tabular}

Pode-se observar através da Tabela 6 que as misturas desfosforantes que apresentaram melhor eficiência de desfosforação foram as misturas M2 e M3, com valores de $69,74 \%$ e $69,23 \%$ respectivamente. Essas misturas desfosforantes tem massa de $1250 \mathrm{~kg}$ e $1000 \mathrm{~kg}$, as maiores massas de misturas usadas no presente trabalho, o que é um indicativo de que maiores massa de desfosforante favorecem melhor eficiência do processo. Também é possível observar que o aumento de massa específica de desfosforante adicionado (massa de desfosforante/ massa de gusa) aumentou a eficiência do processo de desfosforação (valores acima de 42,66 $\mathrm{kg} / \mathrm{t}$ obtiveram maiores eficiências).

A simulação mostrou uma porcentagem de líquido de $94,11 \%$ e atividade de wustita de 0,645 , valores aceitáveis para melhores eficiências de desfosforação quando atividade de $\mathrm{CaO}$ seja igual a 1 e se mantenha por todo o processo. Entretanto não houve a formação de $\mathrm{CaO}$ sólido, influenciando de forma prejudicial o valor do Fator de Desfosforação, uma vez que sua formação é importante para que mantenha a escória líquida saturada em $\mathrm{CaO}$ até o fim do processo. Somente formou-se a seguinte fase sólida: $\mathrm{MgO}$ periclase conforme a porcentagem de sólido indicada na Tabela 7.

De acordo com os valores de Fator de Desfosforação encontrados na literatura, o valor obtido para as misturas usadas no presente trabalho é menor. Isso é um indicativo de que a composição das misturas de desfosforantes utilizadas neste trabalho podem ser reavaliadas visando melhor valor para o Fator de Desfosforação, logo, maior eficiência do processo de desfosforação.

Algumas relações podem ser avaliadas a partir da Tabela 6 , conforme visualizado nas Figuras 4 (a), (b), (c). 

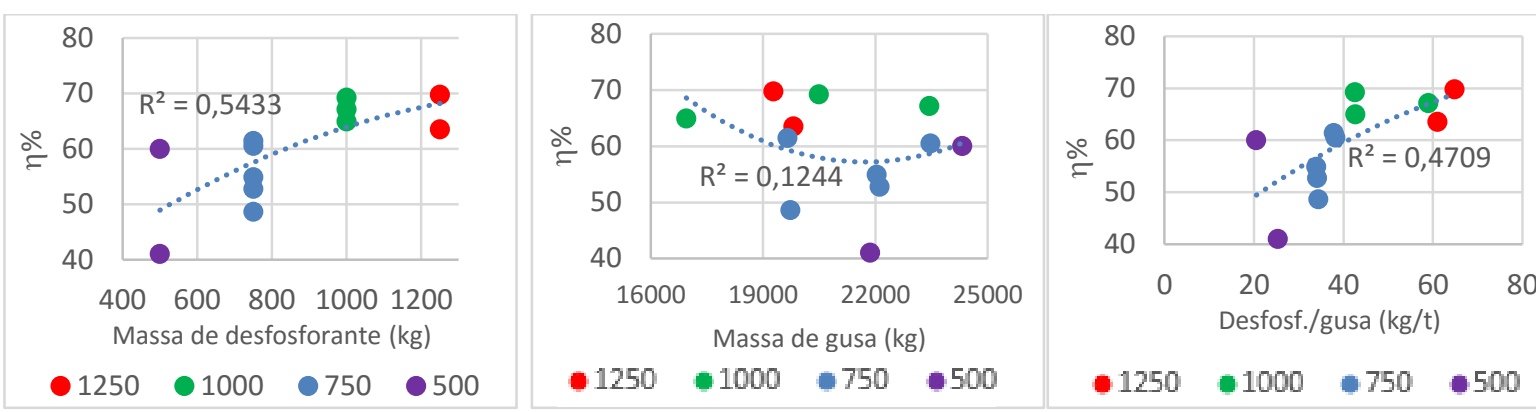

Figura 4. Relação entre a eficiência e (a) massa de desfosforante, (b) massa de gusa, (c) razão massa de desfosforante e massa de gusa.

De acordo com a Figura 4 (a) observa-se que as misturas desfosforantes com maior massa (1000 kg e $1250 \mathrm{~kg}$ ), M2 e M3 respectivamente, apresentaram melhor eficiência de desfosforação. A partir da Figura 4 (b) pode-se analisar que a massa de gusa não atua como um bom indicativo de eficiência do processo de desfosforação quando como um parâmetro isolado, uma vez que para melhores valores de eficiência de desfosforação há grande variação das massas de gusa. Todavia a Figura 4 (c) apresenta uma boa relação entre a eficiência do processo e a razão entre a massa de desfosforante e massa de gusa para as misturas de desfosforante de $1000 \mathrm{~kg}$ e $1250 \mathrm{~kg}$ (M1, M2, M3, M4, M7). Verifica-se uma tendência de maior eficiência de desfosforação quando há um aumento da razão de massa de desfosforante e massa de gusa, de forma que ambos os parâmetros devem ser analisados juntos visando um ponto ótimo para eficiência do processo desfosforante.

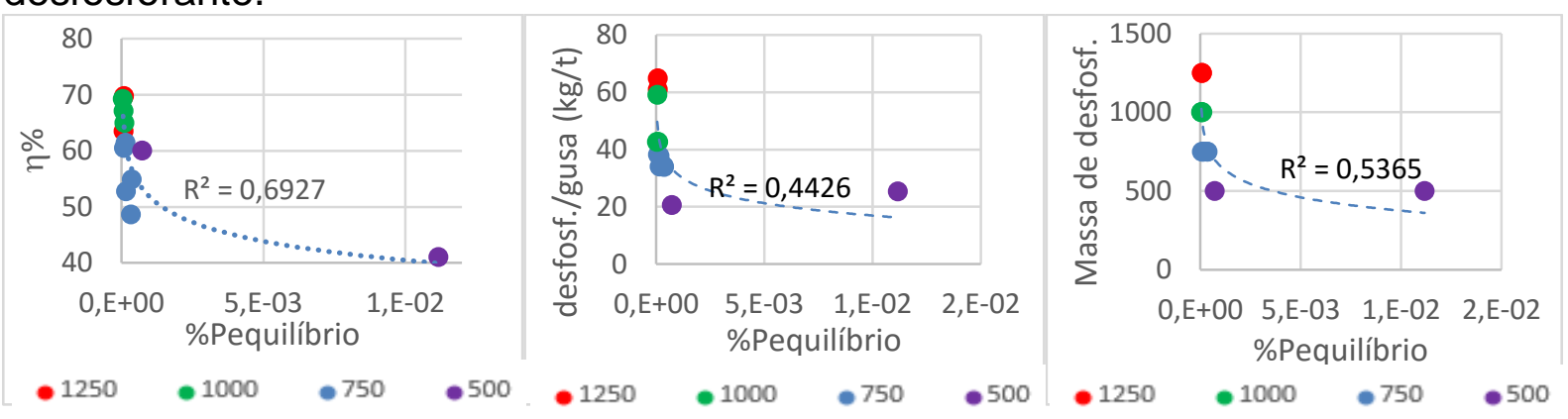

Figura 5. Relação entre a porcentagem de fósforo de equilíbrio e (a) a eficiência, (b) razão massa de desfosforante e massa de gusa e (c) massa de desfosforante.

As misturas desfosforantes M3 e M2 com maiores massas, $1000 \mathrm{~kg}$ e $1250 \mathrm{~kg}$ respectivamente, e que obtiveram as melhores eficiências de desfosforação foram as que apresentaram os menores valores encontrados de fósforo de equilíbrio conforme pode ser visualizado na Figura 5 (a), (b) e (c).

Da Figura 5 (a), pode-se analisar que para a mistura de desfosforante M11 cuja eficiência de desfosforação foi de $41,026 \%$, o menor valor dentre as misturas usadas no presente trabalho, o fósforo de equilíbrio foi aproximadamente 200 vezes maior que o da mistura de desfosforante M3 cujo valor de fósforo de equilíbrio foi o menor obtido, e aproximadamente 125 vezes maior que o da mistura desfosforante M2, a qual apresentou melhor eficiência de desfosforação.

A partir da Figura 5 (b) observa-se que a mistura M8 cuja razão massa de desfosforante e massa de gusa foi a menor, o fósforo de equilíbrio obtido foi aproximadamente 8 vezes maior que o da mistura de desfosforante $M 2$, tendo a mistura M2 valores de razão de massa de desfosforante e massa de gusa 3 vezes maior que M8, e a eficiência de desfosforação para M2 foi o melhor obtido. Por essa 
figura, observa-se que as misturas com menor fósforo de equilíbrio obtidos, foram as misturas que apresentaram a razão de massa de desfosforante e massa de gusa entre 60 a $40 \mathrm{~kg}$.

Através da figura 5 (c) avalia-se que as misturas com maiores valores de massa de desfosforante utilizados permitiram obter menores valores de fósforo de equilíbrio.

\section{CONCLUSÃO}

Considerando os objetivos propostos neste trabalho em paralelo aos resultados obtidos, pôde-se chegar as seguintes conclusões:

- Verificou-se uma tendência de maior eficiência de desfosforação quando há um aumento da razão de massa de desfosforante e massa de gusa (massa específica de desfosforante);

- Os parâmetros termodinâmicos calculados, coeficiente de partição do fósforo (LP), capacidade de fosfato $\left(C_{P}\right)$ e basicidade ótica $(\Lambda)$ não são precisos para prever a eficiência do processo de desfosforação uma vez que se tratam do equilíbrio termodinâmico;

- De acordo com os valores de Fator de Desfosforação encontrados na literatura, o valor obtido para as misturas usadas no presente trabalho é menor. O que pode ser explicado pelo fato de que não houve a formação de $\mathrm{CaO}$ sólido, influenciando de forma prejudicial o valor do Fator de Desfosforação;

- As misturas desfosforantes com maiores massas (1000 e 1250kg) e que obtiveram as melhores eficiências de desfosforação foram as que apresentaram os menores valores encontrados de fósforo de equilíbrio;

- A massa ideal de desfosforante para o processo analisado estaria entre $1000 \mathrm{~kg} \mathrm{e}$ $1250 \mathrm{~kg}$ uma vez que foi observado melhores valores de redução do teor de $\mathrm{P}$.

\section{Agradecimentos}

À FAPES pela bolsa de mestrado.

\section{REFERÊNCIAS}

1 BROSEGHINI, F.C. Utilização da termodinâmica computacional no estudo da desfosforação de ferro-gusa por escórias do Sistema $\mathrm{CaO}-\mathrm{FeO}$. Dissertação de mestrado. Instituto Federal Espírito Santo - IFES, 2015.

2 MUKHERJEE, T.; CHATTERJEE, A. Production of low phosphorus steels from high phosphorus Indian hot metal: Experience at Tata Steel. Bulletin of Material Science, n. 6, v. 19, p. 893-903, 1996.

3 CAMPOS, V. F. Curso sobre Pré-refino de Ferro-Gusa. 1. ed. Belo Horizonte: Universidade Federal de Minas Gerais, 1984. 31p.

4 TURKDOGAN, E.T. Fundamentals of Steelmaking. 1. ed. London: The Institute of Materials, 1996. 331p.

5 HEALY, G. A New Look at Phosphorus Distribution. Journal of the Iron and Steel Institute, n. 6, v. 208, p. 664-668, 1970.

6 WEI, P.; SANO, M.; HIRASAWA, M.; MORI, K. Kinects of Phosphorus Transfer between Iron Oxide Containing Slag and Molten Iron of High Carbon Concentration under Ar-O2 Atmosphere. ISIJ International, n. 4, v. 33, p. 479-487, 1993.

7 HINO, Y.; KIKUCHI, N.; NABESHINA, S.; TAKEUCHI, S. Effect of iron oxide feeding rate and hot metal temperature on dephosphorization rate in torpedo car. ISIJ International, 827-834, 2005.

8 SAMPAIO, R. A. Utilização dos softwares de termodinâmica computacional no estudo das etapas de pré-tratamento do ferro-gusa. Dissertação de Mestrado. Insituto Federal Espírito Santo - IFES, 2014. 